E-187 COMPARATIVE ANALYSIS OF PROCEDURE RELATED MORBIDITY AND MORTALITY BETWEEN PIPELINE, PIPELINE FLEX EMBOLIZATION DEVICE AND SURPASS

${ }^{1} \mathrm{C}$ Feigen, ${ }^{1} \mathrm{~N}$ Haranhalli, ${ }^{1} \mathrm{R}$ Holland, ${ }^{2} \mathrm{~A}$ Brook, ${ }^{1} \mathrm{D}$ Altschul ${ }^{*} .{ }^{1}$ Neurosurgery, Montefiore Medical Center, Bronx, NY; ${ }^{2}$ Neuroradiology, Montefiore Medical Center, Bronx, NY

10.1136/neurintsurg-2020-SNIS.218

Introduction The purpose of this study is to compare peri-procedural complication rates in patients at a single academic medical center treated with Surpass or Pipeline/Pipeline Flex flow diverter stents.

Methods We performed a retrospective chart review of patients who underwent placement of a flow diverter stent from the last 3 years. We evaluated major complications including mortality, stroke, intracranial hemorrhage $(\mathrm{ICH})$ or failure of device deployment, and minor complications including radial and groin access site hematoma, retroperitoneal hematoma (RPH), arterial dissection. We also recorded hospital course complications including urinary tract infection (UTI), pneumonia (PNA) and deep vein thrombosis (DVT). We examined the length of hospital stay (LOS), and disposition at discharge. We adjusted for the following variables: Patient age, gender, pre-existing medical comorbidities, laterality, anatomic location of treated aneurysm, device utilized, number of devices deployed per case, longest length and diameter of all devices deployed per case.

Results We identified 23 Surpass cases and 99 pipeline cases performed on a total of 106 patients. The median number of devices used for Surpass was 1 (IQR 1,1) compared to Pipeline/Pipeline Flex 1 (IQR 1,3) which was statistically significantly different $(p=0.0003)$ We found no significant difference in mortality $(\mathrm{p}=0.4949)$, stroke $(\mathrm{p}=0.3565)$, intracerebral hemorrhage $(p=0.3310)$, or aborted procedures $(p=0.4021)$. There was no significant difference in minor complications or hospital complications. The median length of stay was 1 day for both devices. Interquartile length for Surpass 1-3(75th\%); and Pipeline 1-26(75th\%), mann-whitney $\mathrm{p}=0.238$ between the medians.

Conclusions These results suggest that Surpass is non-inferior to Pipeline in regards to major and minor peri-procedural complications. Pipeline on average required a statistically significant more number of devices per procedure. There was no difference in the length of stay between Surpass and Pipeline. Further prospective data is needed to further confirm this hypothesis.

Disclosures C. Feigen: None. N. Haranhalli: None. R. Holland: None. A. Brook: None. D. Altschul: 2; C; Microvention, Stryker.

\section{E-188 HOW FAR CAN WE GO? - WEB TECHNOLOGY FOR THE TREATMENT OF SIDEWALL IA. INITIAL EXPERIENCE IN A SINGLE INSTITUTION}

B Pabon Guerrero*, J Gutierrez Banos, M Patino Hoyos, V Torres, L Moreira Ponce, 0 Vargas, J Mejia. Neurointevencionismo, AngiTeam, Medellin, Colombia

10.1136/neurintsurg-2020-SNIS.219

Introduction Few years after introduction, Flow disruption technology using WEB device has been used safely for the treatment of wide-neck bifurcation aneurysms, but the use of this endosaccular approach to treat side-wall lesions in terms of feasibility, safety, stability and aneurysm occlusion rate after this treatment is unknown.

Materials and Methods Patients were carefully selected IRB approved. Clinical, anatomical, angiographical and technical considerations were analyzed. Procedure related complications, procedural time, antiplatelet therapy requirements. Web Occlusion Scale (WOS) was used for the Follow-up.

Results From August 2017 and March 2020 a total of six wide-necked, sidewall, IA were selected for WEB treatment. Aneurysm mean size $5.3 \mathrm{~mm}$ in width and 5.8 in height. Aneurysm Location: ICA 3 cases (two Carotid-ophtalmic segment, one AChoA segment), Superior Cerebellar Artery SCA in two patients (33\%), and one impressive case in posterior circulation associated with a basilar fenestration next to VBJ. Four cases were unruptured (66\%), and two cases with history of SAH. DAPT used pre operatively in all cases but none patient remain under antiplatelets after procedure. Technical success of $100 \%$. Mean procedure time: $24 \mathrm{~min}$. None related procedure complications recorded. Immediately angiographic occlusion was evidenced in 3 cases (two SCA and one ICA). Radiological Follow up (ranging 1-26 months) available in $4 / 6$ showed a WOS adequate occlusion in all cases.

Conclusion In our early experience using WEB device to treat different conditions than bifurcation IA's, the results showed that endossacular approach was feasible in highly selected patients, safety profile in agreement with previous bifurcation experiences and very effective to treat challenge cases with a high probability of recurrence or therapeutic failure. Larger series and controlled studies are required to expand its indications in a near future.

Disclosures B. Pabon Guerrero: None. J. Gutierrez Banos: None. M. Patino Hoyos: None. V. Torres: None. L. Moreira Ponce: None. O. Vargas: None. J. Mejia: None.

\section{E-189 SYSTEMATIC REVIEW AND META-ANALYSIS OF VESSEL WALL IMAGING OF INTRACRANIAL ANEURYSMS: A SECOND LOOK}

${ }^{1} \mathrm{~A}$ Larson*, ${ }^{1} \mathrm{G}$ Lanzino, ${ }^{2} \mathrm{~W}$ Brinjikji. ${ }^{1}$ Neurosurgery, Mayo Clinic, Rochester, MN: ${ }^{2}$ Radiology, Mayo Clinic, Rochester, MN

10.1136/neurintsurg-2020-SNIS.220

Our group has previously performed a systematic review and meta-analysis regarding the utility of vessel wall imaging with high-resolution magnetic resonance imaging which demonstrated an association between intracranial saccular aneurysm wall enhancement and aneurysm instability. Given the likely increased utilization of vessel wall imaging technology in the time since our previous review, we sought to perform an updated analysis of the association between aneurysm wall enhancement and unstable intracranial aneurysms. This study was performed according to Preferred Reporting Items for Systematic reviews and Meta-Analyses guidelines. Eligible studies were identified through a comprehensive literature review. A meta-analysis was conducted to examine the association between aneurysm wall enhancement and aneurysm instability by using a random-effects model. Seven studies constituting 728 saccular aneurysms in 564 patients were included. Aneurysms that showed vessel wall enhancement had statistically significant higher odds of being unstable (odds ratio [OR]: 10.7; $95 \%$ confidence interval [CI]: 6.8-16.8; $\left.\mathrm{I}^{2}: \quad 64.3 \%\right)$. The 